\title{
Optical valley Hall effect for highly valley- coherent exciton-polaritons in an atomically thin semiconductor
}

Nils Lundt ${ }^{1}$, Łukasz Dusanowski ${ }^{1}$, Evgeny Sedov ${ }^{2,3}$, Petr Stepanov ${ }^{4}$, Mikhail M. Glazov ${ }^{5}$, Sebastian Klembt $^{1}$, Martin Klaas ${ }^{1}$, Johannes Beierlein ${ }^{1}$, Ying Qin ${ }^{6}$, Sefaattin Tongay ${ }^{6}$, Maxime Richard ${ }^{4}$, Alexey V. Kavokin $^{7,8,2}$, Sven Höfling ${ }^{1,9}$ and Christian Schneider ${ }^{1}$

${ }^{1}$ Technische Physik and Wilhelm-Conrad-Röntgen-Research Center for Complex Material Systems, Universität Würzburg, D-97074 Würzburg, Am Hubland, Germany

${ }^{2}$ Physics and Astronomy School, University of Southampton, Highfield, Southampton, SO171BJ, UK

${ }^{3}$ Vladimir State University named after A.G. and N.G. Stoletovs, Gorky str. 87, 600000, Vladimir, Russia

${ }^{4}$ Univ. Grenoble Alpes, CNRS, Grenoble INP, Institute Neel, 38000 Grenoble, France

${ }^{5}$ Ioffe Institute, Polytechnicheskaya 26, 194021 St. Petersburg, Russia

${ }^{6}$ Arizona State University, Tempe, Arizona 85287 USA

${ }^{7}$ Westlake University, 18 Shilongshan Road, Hangzhou 310024, Zhejiang Province, China

${ }^{8}$ Institute of Natural Sciences, Westlake Institute for Advanced Study, 18 Shilongshan Road, Hangzhou 310024, Zhejiang Province, China

${ }^{9}$ SUPA, School of Physics and Astronomy, University of St. Andrews, St. Andrews KY 16 9SS, United Kingdom

Spin-orbit coupling is a fundamental mechanism that connects the spin of a charge carrier with its momentum. Likewise, in the optical domain, a synthetic spin-orbit coupling is accessible by engineering optical anisotropies in photonic materials. Both yield the possibility to create devices directly harnessing spin- and polarization as information carriers. Atomically thin transition metal dichalcogenides (TMDC) promise intrinsic spin-valley Hall features for free carriers, excitons, as well as photons. Here we demonstrate spin and valley selective propagation of exciton-polaritons in a monolayer of $\mathrm{MoSe}_{2}$ strongly coupled to a microcavity photon mode. In a wire-like device we trace the flow, and the helicity of exciton-polaritons expanding along its channel. By exciting a coherent superposition of $K$ and $K^{\prime}$ tagged polaritons, we observe valley selective expansion of the polariton cloud without neither external magnetic field nor coherent Rayleigh scattering. The observed optical valley Hall effect (OVHE) occurs on a macroscopic scale offering the potential for applications in spinvalley locked photonic devices. 
Spin-valley locking is a striking feature of free charge carriers and excitons emerging in monolayers of transition metal dichalcogenides (TMDCs) ${ }^{1,2}$. It originates from the strong spin-orbit interaction ${ }^{3}$, which arises from the heavy transition metals in TMDCs and the broken inversion symmetry of the crystal lattice. This leads to inverted spin orientations at opposite $\mathrm{K}$ points at the corners of the hexagonal Brillouin zone, for both conduction band electrons and valence band holes. As a result, the $\mathrm{K}$ and $\mathrm{K}^{\prime}$ valleys can be selectively addressed by $\sigma^{+}$and $\sigma^{-}$circularly polarized light ${ }^{4,5}$, which is referred to as valley-polarization. Likewise, coherent superpositions of both valleys can be excited by linear polarized light, which is referred to as valley coherence. The outstanding control of the valley pseudospin has attracted great interest in exploiting this degree of freedom to encode and process information by manipulating free charge carriers ${ }^{6}$ and excitons $s^{7,8,9}$, which has led to the emerging field of valleytronics ${ }^{10}$. However, exciton spin-valley applications are strongly limited by the depolarization mechanisms due to the strong Coulomb exchange interaction of electrons and holes, as well as by the limited exciton diffusion and propagation lengths.

Thus far, in most experiments, TMDC monolayers were non-resonantly excited several hundreds of millielectronvolt above the ground state, so that excitons were created with a finite center of mass wave vector that deviates from the quasi-momentum of the $\mathrm{K} / \mathrm{K}^{\prime}$ valley ${ }^{11}$. In this configuration, the long-range Coulomb exchange interaction of the exciton's constituent electron and hole creates an efficient depolarization mechanism ${ }^{12,13}$. The eigenstates of excitons with their dipole orientation parallel to the wave vector (longitudinal exciton) and with their dipole orientation perpendicular to the wave vector (transverse exciton) are split in energy. This so called L-T splitting increases linearly with the center of mass wave vector, and mixes the $K$ and $K^{\prime}$ excitons, which ultimately leads to a depolarization of excitons during their energy relaxation ${ }^{14}$.

One way to reduce this depolarization effect, is to excite the system quasi-resonantly close to the exciton resonance energy. As a result, excitons are created with small in-plane wave vectors, corresponding to a relatively small L-T splitting and they need to undergo less inelastic scattering events before their radiative decay as compared to non-resonantly excited excitons. This approach has been successively used to measure reasonably high degrees of circular polarization (DOCP) in $\mathrm{MoS}_{2}$ $(40 \%)^{15}, \mathrm{WS}_{2}(90 \%)^{16}$ and $\mathrm{WSe}_{2}(40 \%)^{17}$ monolayers at cryogenic temperature and DOCP values up to $30 \%$ in a $\mathrm{WS}_{2}$ monolayer a room temperature ${ }^{18}$. However, in $\mathrm{MoSe}_{2}$ only a few percent DOCP has been observed this way ${ }^{19,20}$. To achieve close-to-unity valley polarization, excitons in $\mathrm{WSe}_{2}$ have been resonantly excited in a two-photon absorption process ${ }^{21}$.

An alternative approach to enhance the valley polarization is the tailoring of the electrodynamic environment of the monolayer. This can be accomplished by integration of a TMDC monolayer into an optical microcavity, resulting in the formation of exciton-polaritons (polaritons) 20,22 . This approach has been used in particular to increase the DOCP in $\mathrm{MoS}_{2}$ and $\mathrm{WS}_{2}$ monolayers at room temperature $\mathrm{e}^{23-25}$ and to induce a significant valley polarization in $\mathrm{MoSe}_{2}$ monolayer ${ }^{20,26}$ at cryogenic temperatures. Here, 
the strong coupling between the optical cavity mode and the monolayer exciton speeds up the energy relaxation and decay dynamics, which is beneficial for preserving the valley polarization ${ }^{20,23,26}$. Moreover, inter-valley scattering is greatly suppressed in the strong coupling regime ${ }^{20}$, owing to the strongly reduced disorder scatting of the photon component of the polariton ${ }^{27}$. In addition, polaritons in TMDC monolayers have a much longer diffusion length as compared to excitons in a bare monolayer ${ }^{28-30}$, which should be a great advantage with respect to spatial separation of valley tagged excitations.

In this work, we take advantage of these two approaches to enhance valley polarization and valley coherence in a TMDC monolayer. First, we strongly couple excitons in an elongated monolayer $\mathrm{MoSe}_{2}$ to the optical mode of a high quality factor mechanically assembled microcavity. Next, we excite the exciton-polariton states quasi-resonantly close to their ground state by a two-photon absorption process. Combining both approaches enables us to generate valley polarization and valley coherence of the quasi-particles with circular and linear polarization degrees of more than $90 \%$. The ultimate control over valley polarization and valley coherence is a key to observe the optical valley Hall effect. In this effect the polaritons with opposite valley indices propagate in different directions resulting in the valley separation of exciton-polaritons, alike spin-up and spin-down electrons in a semiconductor driven by an electric field separate ${ }^{31}$. We evidence this effect by studying the polarization of polaritons expanding along the monolayer. The spin-Hall phenomenology in optical distributed Bragg reflector (DBR) cavities, which is translated to the valley Hall physics in chiral valley monolayers, arises from the splitting of the transverse electric (TE) and transverse magnetic (TM) modes at finite in-plane wave vectors $^{32}$. This is qualitatively similar to the excitons' L-T splitting, but the TE-TM splitting is approximately two orders of magnitude larger ${ }^{27}$. Thus, if excitons strongly couple to a microcavity photon mode and propagate with finite velocities, the emergent polaritons experience a spindependent propagation ${ }^{32}$. In our sample, this is manifested in a clearly observed and well controllable spatial separation of valley tagged polaritons, which is a clear-cut feature of the OVHE ${ }^{27}$.

\section{Sample structure and polariton formation}

The studied sample structure is schematically depicted in Fig. 1a. The mechanically assembled microcavity is built by transferring a $\mathrm{MoSe}_{2}$ monolayer with a dry-gel method ${ }^{33}$ onto a $\mathrm{SiO}_{2} / \mathrm{TiO}_{2}$ bottom DBR (10 pairs, stop band center at $750 \mathrm{~nm}$ ). The monolayer was mechanically exfoliated from a bulk crystal. Subsequently, we spin-coated a $126 \mathrm{~nm}$ thick poly-methyl-methacrylate (PMMA) buffer layer. Finally, a piece of a separate $\mathrm{SiO}_{2} / \mathrm{TiO}_{2} \mathrm{DBR}$ with lateral dimensions of a few tens of $\mu \mathrm{m}$ (8.5 pairs, stop band center at $750 \mathrm{~nm}$ ) is mechanically peeled off its substrate. This piece is transferred onto the buffer layer with the same dry-gel method ${ }^{33}$. Van der Waals forces are sufficiently strong to keep the top DBR in place. We found that such microcavities demonstrate Q-factors up to 4600 (see supplementary section S1). Figure $1 \mathrm{~b}$ shows a microscope image of the final structure (top view), where the $\mathrm{MoSe}_{2}$ monolayer $(8 \times 40 \mu \mathrm{m})$ is marked in blue. A photoluminescence $(\mathrm{PL})$ spectrum of the monolayer capped 
with PMMA was taken at $5 \mathrm{~K}$ (Fig. 1c) before the microcavity was completed with the top DBR. The monolayer exhibits a neutral exciton resonance at $745.5 \mathrm{~nm} / 1.663 \mathrm{eV}$ and a charged exciton (trion) resonance at $759.8 \mathrm{~nm} / 1.632 \mathrm{eV}$. Figure $1 \mathrm{c}$ also shows a reflectivity spectrum of the uncoupled cavity mode at $747.3 \mathrm{~nm} / 1.659 \mathrm{eV}$, measured in the close-to-the-monolayer position.

First, we probe the in-plane-momentum-resolved PL of the full structure at the monolayer position at $5 \mathrm{~K}$ under continuous-wave excitation with a titanium-sapphire laser (Msquared Solstis) at $740 \mathrm{~nm}$. Neither the cavity mode, nor exciton or trion mode can be observed but a new mode appears with a ground state at $761.4 \mathrm{~nm} / 1.628 \mathrm{eV}$, as presented in Fig. 1d. This mode has a parabolic dispersion at small in-plane momentum components parallel to the long axis of the monolayer. In contrast, if the dispersion is measured perpendicular to the monolayer extension, discrete levels appear in the PL spectrum as depicted in Fig. 1e. The mode splitting of $2.7 \mathrm{meV}$ between the fundamental and first excited mode is fully consistent with an optical mode confinement caused by the monolayer width of $8 \mu \mathrm{m}$ (see supplementary section S5). We attribute this new mode to the lower polariton branch of the strongly coupled exciton-cavity system. The upper polariton branch, and likewise, lower polariton states with large in-plane wave vectors, are typically not observed in photoluminescence spectra acquired under non-resonant pumping, since the thermal population of polaritons can be very low at cryogenic temperatures ${ }^{34}$. In order to provide further evidence for the formation of exciton-polaritons, i.e. the strong light-matter coupling regime, we have carried out two-photon excitation experiments (supplementary section S2), magnetic field measurements (supplementary section S3) and white-light reflection measurements at various temperatures (see supplementary section S4). Based on these measurements, we have modelled the presented lower polariton dispersion by a two-coupledoscillator model, which yield a normal mode splitting of $46 \mathrm{meV}$ and an exciton fraction of $26 \%$ in the ground state (see supplementary section S2). The two-coupled-oscillator model is also presented as an overlay to the measured dispersion in Fig. 1 d.

\section{Polariton valley polarization and valley coherence}

TMDC monolayers can be excited under two-photon absorption ${ }^{21}$, which originates from a two-photon interband transition and also gives rise to the second harmonic generation (SHG) process ${ }^{35,36}$. This strong non-linear response is transferred to our strongly coupled system ${ }^{37}$ and thus provides an ideal setting to study polaritons under nearly resonant excitation conditions. This allows us to directly address polaritons arising from specific valleys of the embedded crystals via quasi-resonant chiral twophoton absorption. The clean, high-fidelity excitation of valley polaritons is compulsory for studies of exciton-polaritons in valleytronic experiments. Here, we excite our system at $5 \mathrm{~K}$ with 2 ps laser pulses at $1518 \mathrm{~nm}$ (Coherent Mira-OPO system, $76 \mathrm{MHz}$ repetition rate) with $\sigma^{+}$and $\sigma^{-}$circular polarization and analyze the polarization of the emitted light (Fig. 2a). The measured spectra for $\sigma^{+}$and $\sigma^{-}$ excitations are plotted in Figs. $2 \mathrm{~b}$ and c, respectively. We observe that the emitted signal is strongly 
polarized with an opposite helicity as compared to the polarization of excitation. Observing a counterrotating signal is fully consistent with SHG selection rules for crystals with broken inversion symmetry and a three-fold rotation symmetry ${ }^{38}$, and likewise for two-photon active interband transitions ${ }^{21,35}$. In Figs. $2 b$ and $2 c$, we plot the degree of circular polarization of the emission across the acquired spectrum, including genuine second harmonic signals and luminescence following the two-photon absorption process (more details on the distinction of SHG vs. two-photon-induced photoluminescence (2P-PL) are given in supplementary sections S6, S7 and S9). We find that a valley polarization of more than $90 \%$, as demonstrated by the corresponding DOCP, is preserved even at energies that are notably below the second harmonic resonance, which is characteristic for $2 \mathrm{P}_{-} \mathrm{PL}^{21,36}$. The outstandingly high valley polarization is explained by two factors: first, the excited polariton states are situated close to the ground state, and therefore their in-plane wave vectors are low $\left(<1.5 \mu \mathrm{m}^{-1}\right)$. Hence, the TE-TM splitting of the cavity and the renormalizations of the exciton oscillator strengths in $L$ and $T$ polarizations, which are the two main mechanisms of the depolarization, are small as compared to the case of non-resonant excitation. Second, the polariton lifetime is shortened to about $200 \mathrm{fs}$ by coupling to the cavity (see supplementary section S8) as compared to the pure excitons in $\mathrm{MoSe}_{2}$ monolayer $\left(390 \mathrm{fs}^{39}\right.$ ). As assessed in Refs. ${ }^{27,20}$, the strong coupling conditions further mitigate the influence of strong exciton localization and disorder scattering, since the spatial extent of the polaritonic wavefunction is much larger than typical excitonic disorder in the monolayer. This enables valley-polariton relaxation timescales that are extended by a factor of approximately 20 . In particular, the negative detuning of the exciton and cavity photon mode is crucial for such suppression of disorder scattering ${ }^{27}$. Thus, a strong increase in the degree of circular polarization can be expected in our resonantly driven cavity polariton system. The resulting value of DOCP is governed by the interplay between radiative decay time $\tau$ and spin-valley relaxation time $\tau_{s}$ and can be approximated by $\rho_{\text {circ }}=$ $\rho_{0} /\left(1+\frac{\tau}{\tau_{s}}\right)^{11,24}$, where $\rho_{0}$ is the initial polarization (close to $100 \%$ ). The spin-valley relaxation time for the pure $\mathrm{MoSe}_{2}$ monolayer exciton is $150 \mathrm{fs}^{20,39}$, resulting in a polariton spin-valley relaxation time of $3 \mathrm{ps}^{20}$. With this, we calculate a valley polarization of $94 \%$, which is in excellent agreement with our experimental observations.

While valley-polaritons can be generated by exciting with circularly polarized light as shown above, excitation with linearly polarized light may induce a coherent superposition of valley polaritons. Valley coherence up to $80 \%$ has been observed in bare monolayers of $\mathrm{MoS}_{2}{ }^{40}, \mathrm{WS}_{2}{ }^{18}$ and $\mathrm{WSe}_{2}{ }^{41}$, but has not been measured in $\mathrm{MoSe}_{2}$ monolayers. For our valley polaritons, the degree of linear polarization (DOLP) $\rho_{\text {lin }}$ of the decaying polariton field provides a direct measure of the dephasing processes of the polariton pseudospin vector on the equator on the Bloch sphere (Fig. 2d). We probe our system by exciting at $1514 \mathrm{~nm}(757 \mathrm{~nm})$ with linearly polarized light in the $X$ - and Y-basis, where the $X$ axis is aligned with one of the crystal axis (see supplementary section S6 and S9 for crystal axis alignment) and the $Y$ axis is perpendicular to the $X$ axis. The emitted signal is subsequently measured in the same 
basis and the DOLP was determined by $\rho_{\text {lin }}=\frac{I(X)-I(Y)}{I(X)+I(Y)}$. Figures $2 \mathrm{e}$ and $2 \mathrm{f}$ show the acquired spectra, along with the calculated DOLP values as a function of the detection wavelength. The polarization of the resonantly scattered fraction of the signal (the SHG part) is expected to be fully governed by the crystal symmetry and yields no information about the valley coherence of the polaritons (see supplementary section S9 for SHG selection rules). However, it is remarkable that a strong linear polarization is maintained in the broad 2P-PL emission of the polariton resonances, even $10 \mathrm{meV}$ below the energy of the SHG signal, with a DOLP clearly exceeding $90 \%$ close to the polariton ground state. Thus, the DOLP clearly reflects the valley coherence.

The phase of the coherent valley superposition can be addressed by changing the direction of the linear polarization of the pump beam. In Fig. 2f, we show that 2P-PL retains the direction of the linear polarization of the pump oriented along the $\mathrm{Y}$-direction, and likewise allows us to extract large degrees of valley coherence exceeding $90 \%$ even 10 meV below the SHG frequency. In contrast, the DOLP signal is close to zero or even negative around the SHG frequency, due to the specific SHG selection rules in TMDC monolayer ${ }^{35}$ (see supplementary section $\mathrm{S} 9$ for details).

While near-unity valley polarization has been observed on bare $\mathrm{WS}_{2}$ monolayers ${ }^{21}$, the demonstrated, unprecedented degrees of valley coherence, in particular in a $\mathrm{MoSe}_{2}$ monolayer, clearly outline the potential of strong light-matter coupling for generation and manipulation of coherent valley superposition states of hybrid light-matter quasiparticles.

\section{Optical Valley Hall Effect}

In contrast to TMDC excitons, which have a diffusion length of a few hundred nanometers in high quality samples 29,30 , TMDC based polaritons are expected to expand over significantly larger distances (on the order of $10 \mu \mathrm{m}$ ) even in the linear, non-ballistic regime due to their small effective masses $^{28}$. Figure $3 a$ depicts the spatial intensity distribution of near-resonantly injected polaritons on the monolayer at $5 \mathrm{~K}$. From this intensity distribution, we can infer that our polaritons propagate by approximately $3.6 \mu \mathrm{m}$ before they decay (see supplementary section S10). It should be noted that an excitation slightly above the LPB (in this case $10 \mathrm{meV}$ ) is essential to create polaritons with finite wave vectors and velocities. Such long propagation lengths allow us to investigate the valley dependent polariton propagation upon valley coherent initialization of the system. This is recorded via spatial- and polarization-resolved luminescence. Here, the real space intensity distribution was measured in $\sigma^{+}$and $\sigma^{-}$basis at $5 \mathrm{~K}$ and the spatial-resolved DOCP was deduced. Figure $3 \mathrm{~b}$ depicts a DOCP distribution recorded under linearly polarized two-photon excitation at $1514 \mathrm{~nm}$. The initial polarization angle was $-75^{\circ}$ with respect to the long monolayer axis. Clearly, the DOCP distribution shows two domains with a left-right separation that is slightly rotated clockwise and features an oscillating pattern along the vertical stripe direction. As we rotate the initial polarization orientation 
by $-45^{\circ}$ (Fig. 3c), we observe a dramatic change in the polarization pattern with a vertical domain separation and weak $\sigma^{+}$regions along the right monolayer edge. We note that similar patterns have been predicted ${ }^{32}$ and observed ${ }^{42,43}$ as spin domains of exciton-polaritons in GaAs QWs embedded in microcavities, known as the optical spin Hall effect ${ }^{32,42}$. In contrast to the GaAs case, the spin and valley indices cannot be treated separately in TMDC monolayer excitons, and consequently, the valley-dependent expansion of polaritons leads to the emergence of the $\mathrm{OVHE}^{27}$.

Based on the physics of the OVHE effect, the intensity and DOCP distributions were modelled for the measured monolayer geometry and the experimental conditions. The details of the model can be found in the Methods section, as well as supplementary sections S11-S13. The simulated intensity distribution, plotted in Fig. 3d, shows a polariton propagation tail along the monolayer similar to the experimentally observed one. The simulated DOCP distributions, calculated as

$\left(\left|\Psi_{+}\right|^{2}-\left|\Psi_{-}\right|^{2}\right) /\left(\left|\Psi_{+}\right|^{2}+\left|\Psi_{-}\right|^{2}\right)$, for the same initial linear polarization orientations that were used in the experiments are plotted in Fig. $3 e$ and $\mathrm{f}$, respectively.

In the case of the $-75^{\circ}$ orientation, the simulation nicely reproduces the valley separation. As in the experiment, the DOCP is increasing towards the flake edges. Polaritons that decay further away from the excitation spot are associated with higher in-plane wave vectors, which in turn provide higher splittings and stronger effective magnetic fields. Consequently, the precession of the pseudospin, which is equivalent to the polarization Stokes vector, is more pronounced leading to a larger valley polarization. Moreover, the wavy domain separation line is also clearly seen in the simulation. This is explained with interfering polaritons that are reflected at the monolayer edges. The simulation of the rotated initial polarization in Fig. $3 f$ is again in good qualitative agreement with our experimental data. The main, bottom section of the monolayer is strongly dominated by a $\sigma^{-}$domain with small $\sigma^{+}$regions at the right monolayer edge. Further experimental data, which were recorded at intermediate excitation angles, support our assignment to the OVHE, and can be found in the supplementary section S14.

The very good agreement of our experimental data with the theoretical modelling allows us to interpret our experimental data as the first manifestation of the OVHE in a TMDC exciton-polariton system. We note, that our experimentally observed valley polarized domains yield DOCP up to $80 \%$, which itself is a remarkable consequence of the strong valley polarization and valley coherence, which is preserved by our experimental approach (see supplementary section S15 for comparative measurements via linear spectroscopy).

\section{Conclusion}

We have demonstrated that near-unity valley polarization and valley coherence can be achieved under near-resonant two-photon excitation by integrating a TMDC monolayer into a high-Q microcavity. Such high degrees of circular and linear polarization are facilitated by the strong coupling 
conditions, which simultaneously reduce the quasi-particle radiative lifetime and prolong the depolarization time at states close to the polariton ground state. Benefiting from these conditions, we have studied the valley dependent, optically controllable propagation of exciton-polaritons. Clear signatures of polarization domains emerge in the expansion of polaritons, observed in the valleycoherent excitation scheme. This is a clear-cut signature of the optical valley Hall effect, which allows us to observe the interplay of spin, valley, and momentum of our quasi-particles, driven by internal pseudo-magnetic fields.

It is remarkable that the combination of two-photon excitation and cavity electrodynamics allows to reach such a high degree of valley control even in $\mathrm{MoSe}_{2}$ monolayers, which is known to exhibit a rather poor DOCP as bare monolayer ${ }^{20}$. It has been argued that the presence of a low-lying dark exciton state in the tungsten based TMDC materials, which is not subject to the exchange-induced depolarization mechanism, indeed is beneficial for the observation of large degrees of valley polarization and coherence ${ }^{44}$. Thus, we suspect that similar experiments, with even more pronounced degrees of polarization can be conceived based on high quality, tungsten based TMDC layers, potentially also at elevated temperatures. While the OVHE of propagating polaritons may be utilized in valleytronic on-chip applications, the interplay of valley coherent superpositions and photonic spinorbit coupling ${ }^{45}$ could be exploited to harness valley-path entanglement phenomena of propagating quantum wavepackets of polaritons. Likewise, by carefully preparing monolayers with imprinted super-potentials for excitons, similar experiments could be conceived to demonstrate topologically non-trivial expanding polaritons in a similar framework ${ }^{46}$, and even pave the way towards topological lasers in the strong coupling regime ${ }^{47}$ based on TMDC crystals.

\section{Methods}

\section{Experimental Setup}

We used an optical setup in which both spatially (near-field) and momentum-space (far-field) resolved spectroscopy and imaging are accessible. Various laser sources could be coupled into the beampath, including a $532 \mathrm{~nm} \mathrm{CW}$ laser, a wavelength tuneable CW laser (700-980 nm), and a wavelength tuneable system composed of a Titanium Saphire laser ( 2 ps pulse length) which is driving an optical parametric oscillator (tuning range $1100-1600 \mathrm{~nm}$ ). For imaging the sample, as well as for white light reflection spectroscopy, we utilized a thermal light-source. The light is then directed through a microscope objective $(0.65 \mathrm{NA})$ onto the sample, which is mounted in a liquid Helium cryostat (temperature down to $4.2 \mathrm{~K}$ ). Using the objective allows probing a sample with a focus spot diameter of $2 \mu \mathrm{m}$ for the laser excitation and $10 \mu \mathrm{m}$ for the white-light illumination. The re-emitted or reflected 
light is collected by the objective and propagates through a lens system to the entrance plane of the spectrometer. Our setting allows us to access both the spatial, as well as the angular distribution of the emitted signal from the sample. To obtain a spatially resolved signal, the emission from the sample is simply projected onto the imaging plane of the spectrometer in a conventional microscope configuration. In order to switch to the angle resolved configuration, an additional lens is placed into the beam path, where its distance to the back focal plane of the objective is equivalent to its focal length. This configuration is also known as Fourier space imaging, which allows imaging the angular distribution of the emitted light onto the Peltier-cooled CCD camera of the spectrometer. The system's angular resolution is $\sim 0.03 \mu \mathrm{m}^{-1}\left(\sim 0.2^{\circ}\right)$. Additionally, a second closed cycle cryostat providing magnetic fields up to $9 \mathrm{~T}$ and sample temperatures down to $1.8 \mathrm{~K}$, is integrated in the setup. In this setting, the PL is collected through 0.81 NA microscope objective for the magnetic field measurements. Both the real space and Fourier space configurations create an image in the focus plane of the spectrometer system. In the spectrometer, this plane is projected onto the CCD chip with two different options. For the first option, a slit in the entrance plane is closed to cut out a narrow section of the image in y direction. The emitted signal is dispersed by an optical grating with either 150,600 or 1200 lines/mm and energy resolutions of $1.1,0.26$ and $0.11 \mathrm{meV}$ (at $750 \mathrm{~nm}$ ), respectively. Ultimately, the CCD chip acquires a data set which carries the spectral information along the $\mathrm{x}$ axis and either spatial position or emission angle along the y axis. Alternatively, the slit can be completely opened, the grating is aligned to act like a mirror (zero order reflection) and the CCD camera is used to acquire an energyaveraged image of the emission. In this way, the CCD chip captures the full real space or Fourier space image.

To probe the sample with a well defined polarization state, a linear polarizer is placed in the excitation path. Subsequently, either a commercial quarter- or half-wave plate are inserted. In the appropriate orientation, the quarter-wave plate induces a phase shift of $\frac{\pi}{2}$ and converts linearly polarized light to circularly polarized light (and vice versa). The half-wave plate induces a phase shift of $\pi$, which alters the orientation of the linearly polarized light. Since all optical components may in principle affect the polarizations state of the incident light, a nominally nonpolarizing beam-splitter, objective and cryostat glass were used. Finally, a polarimeter (Schaefter-Kirchoff SK010PA) was used to quantify the actual polarization state of the incident light at the position where the sample is placed. In this manner, the orientations of the wave plates can be slightly corrected to compensate for the polarizing effect of the optical components beam splitter. As a result, a degree of circular polarization of more than $99 \%$ in front of the cryostat window can be prepared. In the detection path, the emitted light is analyzed with respect to its polarization. Therefore, a quarter-wave plate is inserted and oriented in a way that $\sigma^{+}$is converted to linear polarized light in $\mathrm{V}$ orientation and $\sigma^{-}$is converted into the $\mathrm{H}$ orientation. Then, a linear polarizer is inserted, which transmits all $\mathrm{V}$ components and blocks all $\mathrm{H}$ components. 
Analogously, the quarter-wave plate is rotated to measure the intensity of the $\sigma^{-}$component. It should be noted that all polarization optics were placed before the first mirror in the detection path, which may have an impact on the polarization state. In addition, the linear polarizer in the detection is always kept at the same orientation since the sensitivity of the spectrometer depends on the polarization of the detected light.

\section{Model}

We describe our system by solving the generalized Gross-Pitaevskii equation for the two-component wavefunction $\Psi_{\sigma}(\mathbf{r}, t)$

$$
\mathrm{i} \hbar \partial_{t} \Psi_{\sigma}=[\hat{E}+V(\mathbf{r}, t)] \Psi_{\sigma}+\frac{\Delta}{2}\left(\hat{k}_{x}-\sigma \mathrm{i} \hat{k}_{y}\right)^{2} \Psi_{-\sigma}+\mathrm{i} \frac{\hbar}{2}\left(R n_{\mathrm{R}}^{\sigma}-\gamma\right) \Psi_{\sigma}+P_{\sigma}(\mathbf{r}, t)
$$

coupled to the rate equation for the density of the spin-resolved reservoir of incoherent excitons:

$$
\partial_{t} n_{\mathrm{R}}^{\sigma}=\zeta\left|P_{\sigma}(\mathbf{r}, t)\right|^{2}-\left[\gamma_{\mathrm{R}}+R\left|\Psi_{\sigma}\right|^{2}\right] n_{\mathrm{R}}^{\sigma},
$$

where $\sigma= \pm 1$ describes either spin-up (+) or spin-down (-) states; $\hat{E}$ is the polariton kinetic energy operator, which reproduces the non-parabolic polariton dispersion in Fig. 1d observed experimentally and fitted using the two-coupled-oscillator model (see supplementary section S2); $V(\mathbf{r}, t)=V_{0}(\mathbf{r})+\alpha\left|\Psi_{\sigma}\right|^{2}+\alpha_{\mathrm{R}} n_{\mathrm{R}}^{\sigma}$ is the effective potential experienced by polaritons. It consists of the stationary confinement potential across the sample $V_{0}(\mathbf{r})$ and the blueshift induced by polaritonpolariton interactions within the condensate and interactions of polaritons with excitons in the incoherent reservoir. $\alpha$ and $\alpha_{\mathrm{R}}$ are the corresponding interaction constants; $\alpha=X^{2} \alpha_{\mathrm{R}}$, where $X^{2}$ is the exciton fraction in the polariton state (to minimize the number of parameters we neglected the effect of the oscillator strength saturation); $\Delta$ is the TE-TM splitting constant, $\hat{\mathbf{k}}=\left(\hat{k}_{x}, \hat{k}_{y}\right)=\left(-\mathrm{i} \square_{x},-\mathrm{i} \square_{y}\right)$ is the quasimomentum operator. The polariton condensate is excited by the non-homogeneous resonant optical pump $P_{\sigma}(\mathbf{r}, t)$. To take into account the inevitable appearance of the reservoir of incoherent excitons under the resonant pumping, we introduce the term $\zeta\left|P_{\sigma}(\mathbf{r}, t)\right|^{2} ; \zeta$ is the dimensional reservoir response constant ${ }^{48} ; R$ describes the stimulated scattering rate from the reservoir to the ground state. $\gamma$ and $\gamma_{\mathrm{R}}$ are the decay rates of polaritons and reservoir excitons, respectively.

For the simulation, we take values of the parameters estimated from the experiment. We take the decay rates as $\gamma=1 / 200 \mathrm{fs}^{-1}, \gamma_{\mathrm{R}}=1 / 390 \mathrm{fs}^{-1}$. The pump energy is resonant to the polariton energy at $k=1.5 \mu \mathrm{m}^{-1}$. The LT-splitting is taken as $\Delta k^{2}=0.75 \mathrm{meV}$ (at the same $\mathrm{k}$ ) typical for such kind of structures $^{26}$. To estimate the interaction constants, we refer to the analyses performed in Refs. $[49,50]$. The exciton-exciton interaction constant is approximated as $\alpha_{\mathrm{R}} \square c E_{\mathrm{b}} a_{\mathrm{B}}^{2}$, where $E_{\mathrm{b}}$ and $a_{\mathrm{B}}$ 
are the exciton binding energy and the Bohr radius, respectively, $c$ is the numerical constant. For the characteristic parameters $E_{\mathrm{b}}=0.5 \mathrm{eV}, a_{\mathrm{B}}=2 \mathrm{~nm}$ and $c=2$, the interaction constants are estimated as $\alpha / X^{2}=\alpha_{\mathrm{R}}=4 \mu \mathrm{eV} \mu \mathrm{m}^{2}$. For the exciton scattering rate $R$, the following approximation holds, following from the Fermi golden rule: $R \propto E_{\mathrm{b}} a_{\mathrm{B}}^{2} / \hbar$, which gives us the estimation $R \square 0.005 \mathrm{ps}^{-1} \mu \mathrm{m}^{2}$.

\section{Additional Informations}

Supplementary information is available in the online version of the paper. Reprints and permission information is available online at www.nature.com/reprints. Correspondence and requests for materials should be addressed to C.S.

\section{Contributions}

N.L. exfoliated, identified and transferred the monolayer and designed and fabricated the microcavity. Q.Y. and S.T. synthesized the bulk crystal. N.L., Ł.D., S.K., M.K., P.S. performed experiments. N.L., S.K., M.K., J.B., M.R., C.S. analyzed and interpreted the experimental data, supported by all co-authors. E.S., M.M.G. and A.V.K. provided the theory. N.L. and C.S. wrote the manuscript, with input from all coauthors. C.S and S.H. initiated the study and guided the work.

\section{Data availability}

The Data that support the findings of this study are available from the corresponding author upon reasonable request.

\section{Competing interests}

The authors declare no competing financial interests.

Correspondence and requests for materials should be addressed to Christian Schneider (christian.schneider@uni-wuerzburg.de). 
a

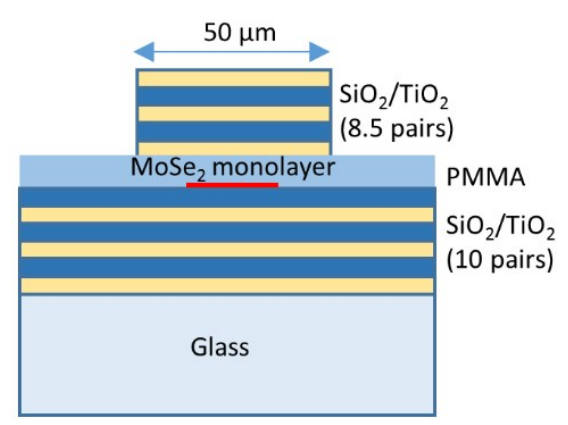

b

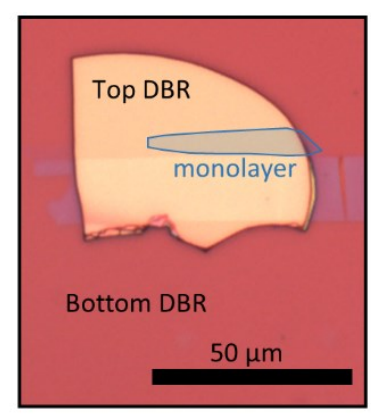

C

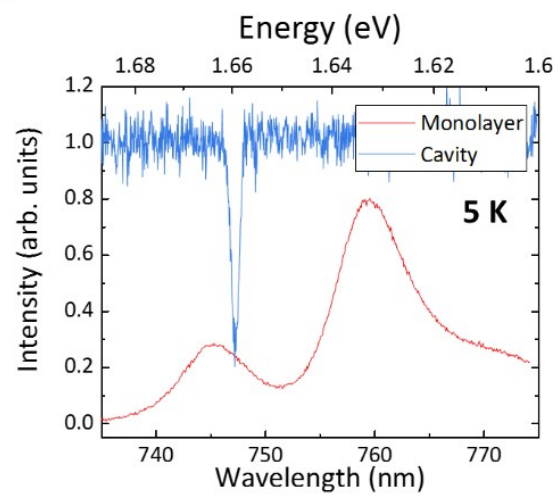

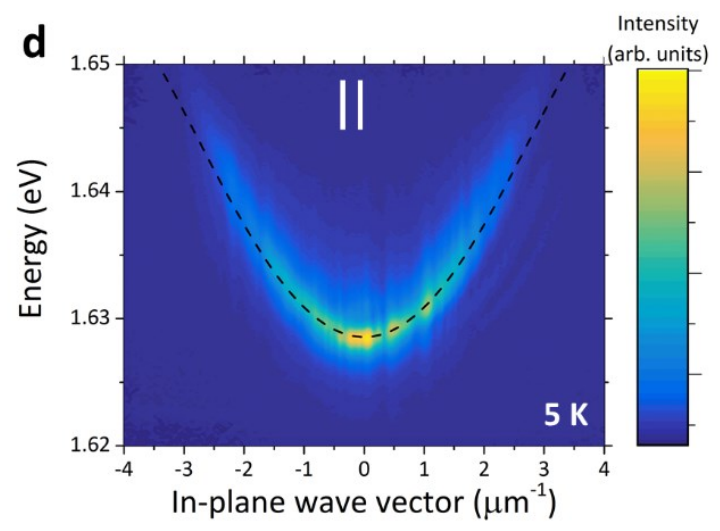

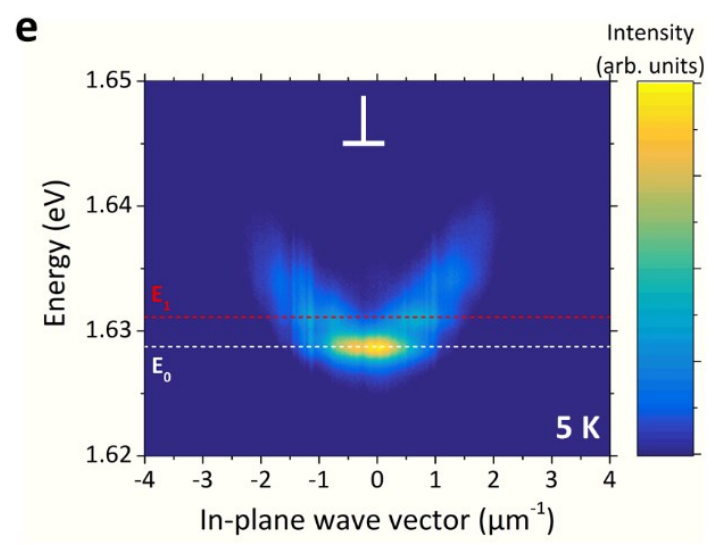

Fig. 1 / Sample design and characterization. a) Sketch of the mechanically assembled microcavity. b) Optical image of the cavity, indicating the elongated monolayer, which provides the channel geometry. c) Photoluminescence measurement of the untreated monolayer and the reflectivity of the optical empty-cavity resonance at $5 \mathrm{~K}$. d) Momentumresolved photoluminescence spectrum recorded at $5 \mathrm{~K}$ along the stripe-direction, as well as perpendicular to the stripe (e). In $d$, the measurement is overlaid with a two-coupled oscillator model, presented in supplementary information S2. In e, the mode discretization, which arises from the finite monolayer width of $8 \mu \mathrm{m}$ is marked for the first (black) and second (red) discretized state. 
a

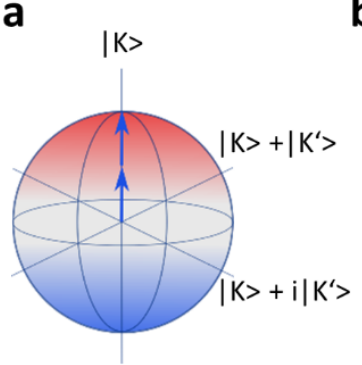

$\mid K^{\prime}>$ b

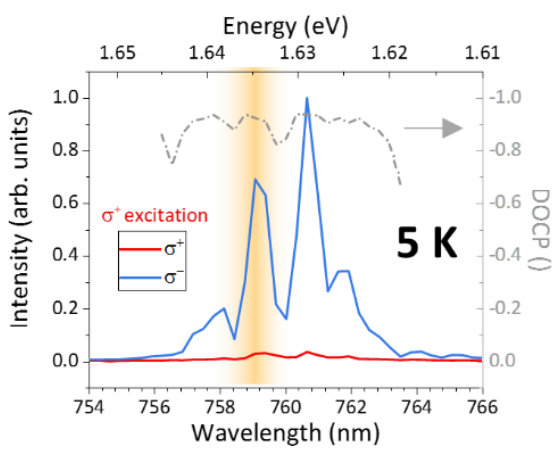

C

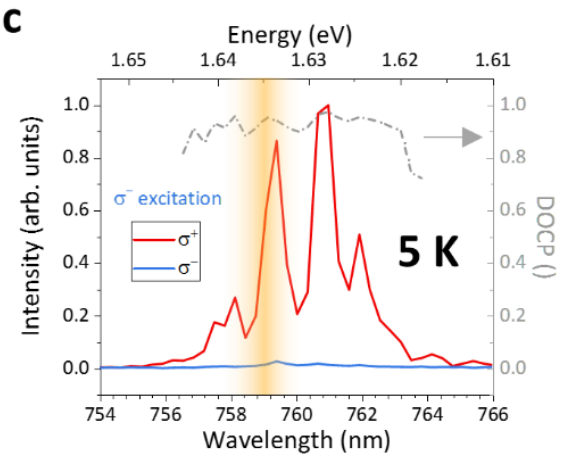

$f$

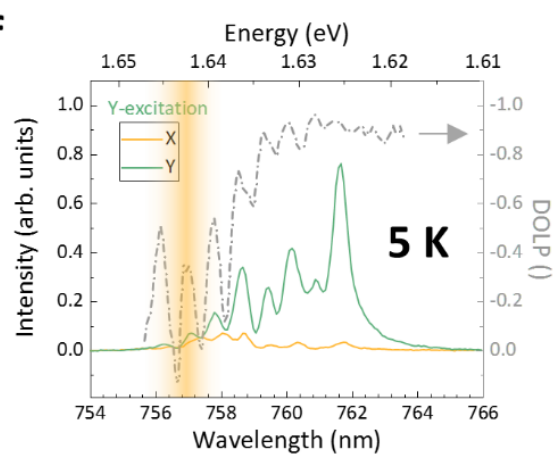

Fig. 2 ( Valley polarization and coherence of polaritons. a) Schematic drawing of the polariton pseudo-spin, which is generated at $K / K^{\prime}$ by quasi-resonant two-photon absorption. b) Polarization-resolved emission spectrum of polaritons, excited at $5 \mathrm{~K}$ by a $\sigma^{+}$polarized nearinfrared laser ( $\sigma^{-}$polarized laser depicted in $\left.c\right)$ ). The degrees of circular polarization of the emitted signals reveal a strongly counter-polarized emission. The energy of the second harmonic resonance is indicated by the orange area. d) Schematic drawing of the excitation of a valley coherent state, generated by quasi-resonant two-photon absorption. e) Polarization-resolved emission spectrum of polaritons, excited at $5 \mathrm{~K}$ with a linearly polarized near-infrared laser in X-basis ( $Y$-basis, linear polarized injection is depicted in $f$ )). The emitted light is strongly co-polarized. The energy of the second harmonic generation resonance is indicated by the orange area. 


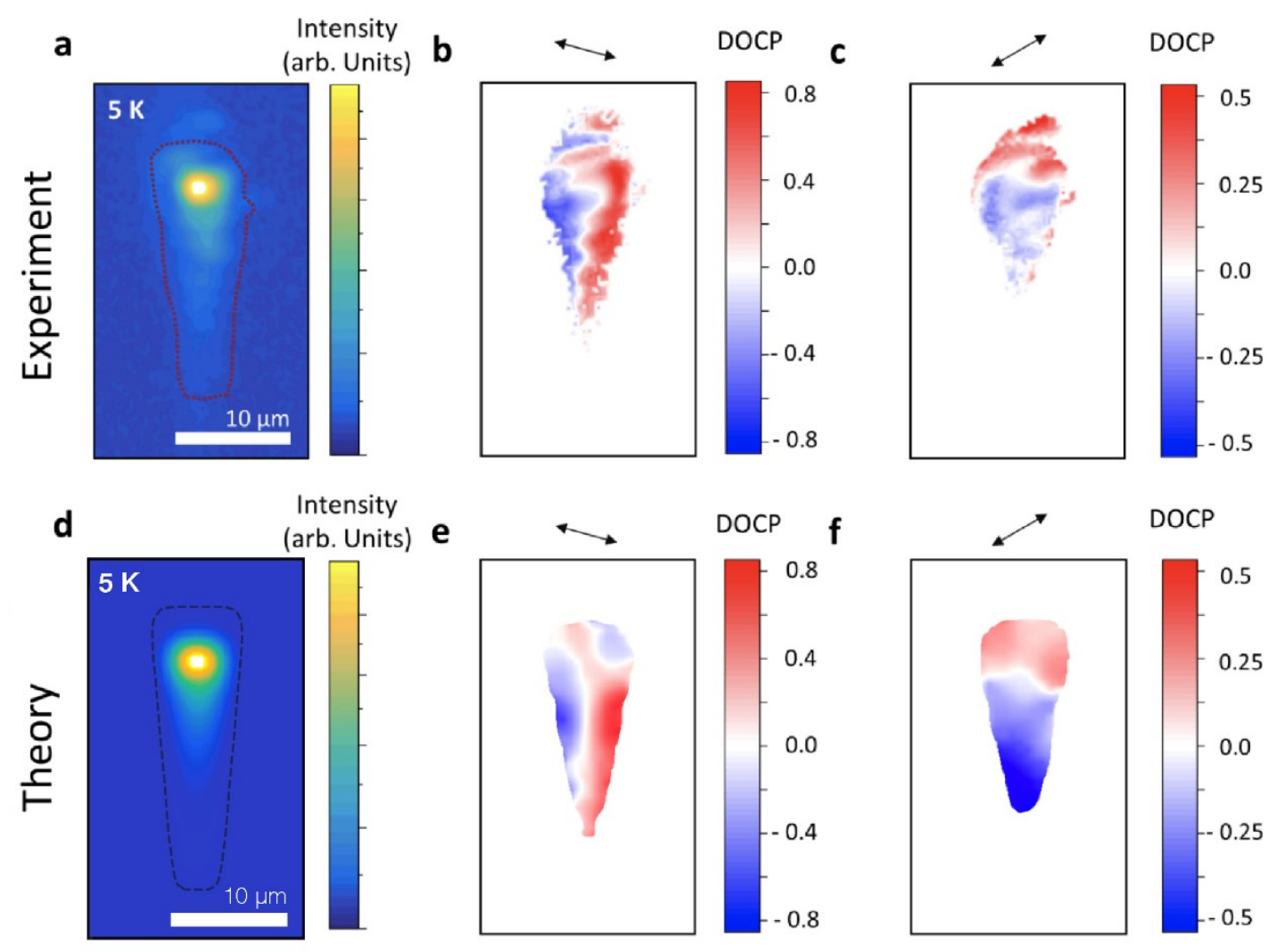

Fig. 3 / Optical Valley Hall Effect. a) PL intensity distribution along the monolayer, observed under two-photon excitation at $1514 \mathrm{~nm}$, where the white point marks the excitation spot on the monolayer. The monolayer edges are indicated by the dotted line. b) Spatially resolved $D O C P$ distribution across the monolayer. The polarization orientation of excitation light (-75。 with respect to the long monolayer axis) is indicated by the double-headed arrow. (c) DOCP distribution across the monolayer with a polarization orientation that was rotated by $-45^{\circ}$ ($120 \circ$ with respect to the long monolayer axis). $d$-f) Simulated intensity and DOCP distributions, each corresponding to the figure above. 


\section{References}

1. Xiao, D., Liu, G. Bin, Feng, W., Xu, X. \& Yao, W. Coupled spin and valley physics in monolayers of MoS 2 and other group-VI dichalcogenides. Phys. Rev. Lett. 108, (2012).

2. $\mathrm{Xu}, \mathrm{X} ., \mathrm{Yao}, \mathrm{W} ., \mathrm{Xiao}, \mathrm{D}$. \& Heinz, T. F. Spin and pseudospins in layered transition metal dichalcogenides. Nat. Phys. 10, 343-350 (2014).

3. Dyakonov, M. I. Spin Physics in Semiconductors. (Springer, 2008).

4. Mak, K. F., He, K., Shan, J. \& Heinz, T. F. Control of valley polarization in monolayer $\mathrm{MoS}_{2}$ by optical helicity. Nat. Nanotechnol. 7, 494-498 (2012).

5. Cao, T. et al. Valley-selective circular dichroism of monolayer molybdenum disulphide. Nat. Commun. 3, 885-887 (2012).

6. Mak, K. F., Mcgill, K. L., Park, J. \& Mceuen, P. L. The valley Hall effect in $\mathrm{MoS}_{2}$ transistors. 344, 75-79 (2014).

7. Onga, M., Zhang, Y., Ideue, T. \& Iwasa, Y. Exciton Hall effect in monolayer $\mathrm{MoS}_{2}$. Nat. Mater. 16, 1193-1198 (2017).

8. Wang, G. et al. Control of Exciton Valley Coherence in Transition Metal Dichalcogenide Monolayers. Phys. Rev. Lett. 117, 187401 (2016).

9. Ye, Z., Sun, D. \& Heinz, T. F. Optical manipulation of valley pseudospin. Nat. Phys. 13, 26-30 (2017).

10. Schaibley, J. R., Yu, H., Yao, W. \& Xu, X. Valleytronics in 2D materials. Nat. Rev. 1, 1-15 (2016).

11. Wang, G. et al. Colloquium : Excitons in atomically thin transition metal dichalcogenides. Rev. Mod. Phys. 90, 21001 (2018).

12. Glazov, M. M. et al. Exciton fine structure and spin decoherence in monolayers of transition metal dichalcogenides. Phys. Rev. B 89, 201302 (2014).

13. Glazov, M. M. et al. Spin and valley dynamics of excitons in transition metal dichalcogenide monolayers. Phys. status solidi 252, 2349-2362 (2015).

14. Maialle, M. Z., Silva, E. \& Sham, L. J. Exciton Spin Dynamics in Quantum-Wells. Phys. Rev. B 47, 15776 (1993).

15. Kioseoglou, G., Hanbicki, A. T., Currie, M., Friedman, A. L. \& Gunlycke, D. Valley polarization and intervalley scattering in monolayer MoS. Appl. Phys. Lett. 101, 221907 (2012).

16. Zhu, B., Zeng, H., Dai, J., Gong, Z. \& Cui, X. Anomalously robust valley polarization and valley coherence in bilayer WS 2 . Proc. Natl. Acad. Sci. 111, 11606-11611 (2014).

17. Jones, A. M. et al. Optical generation of excitonic valley coherence in monolayer WSe $2.8,6-$ 10 (2013). 
18. Hanbicki, A. T. et al. Anomalous temperature-dependent spin-valley polarization in monolayer $W_{2}$. Sci. Rep. 6, 18885 (2016).

19. Wang, G. et al. Polarization and time-resolved photoluminescence spectroscopy of excitons in $\mathrm{MoSe}_{2}$ monolayers. Appl. Phys. Lett. 106, 112101 (2015).

20. Dufferwiel, S. et al. Valley-addressable polaritons in atomically thin semiconductors. Nat. Photonics 11, 497-501 (2017).

21. Seyler, K. L. Electrical control of second-harmonic generation in a $\mathrm{WSe}_{2}$ monolayer transistor. Nat. Nanotechnol. 10, 1-5 (2015).

22. Dufferwiel, S. et al. Valley coherent exciton-polaritons in a monolayer semiconductor Nat. Commun. 9.1, 4797 (2018).

23. Chen, Y., Cain, J. D., Stanev, T. K., Dravid, V. P. \& Stern, N. P. Valley-Polarized ExcitonPolaritons in a Monolayer Semiconductor. Nat. Photonics 11, 431-435 (2017).

24. Sun, Z. et al. Optical control of room-temperature valley polaritons. Nat. Photonics 11, 491496 (2017).

25. Lundt, N. et al. Observation of macroscopic valley-polarized monolayer exciton-polaritons at room temperature. Phys. Rev. B - rapid communciations 96, 241403 (2017).

26. Lundt, N. et al. Valley polarized relaxation and upconversion luminescence from TammPlasmon Trion-Polaritons with a MoSe 2 monolayer. 2D Mater. 4, 25096 (2017).

27. Bleu, O., Solnyshkov, D. D. \& Malpuech, G. Optical valley Hall effect based on transitional metal dichalcogenide cavity polaritons. Phys. Rev. B 96, 165432 (2017).

28. Barachati, F. et al. Interacting polariton fluids in a monolayer of tungsten disulfide. Nature Nanotechnology 13.10, 906 (2018).

29. Kulig, M. et al. Exciton Diffusion and Halo Effects in Monolayer Semiconductors. Phys. Rev. Lett. 120, 207401 (2018).

30. Cadiz, F., Paget, D., Urbaszek, B. \& Marie, X. Exciton diffusion in $\mathrm{WSe}_{2}$ monolayers embedded in a van der Waals heterostructure. Appl. Phys. Lett. 112, 152106 (2018).

31. Dyakonov, M. I. \& Perel, V. I. Current-induced spin orientation of electrons in semiconductors. Phys. Lett. 35, 1-2 (1971).

32. Kavokin, A., Malpuech, G. \& Glazov, M. Optical Spin Hall Effect. Phys. Rev. Lett. 95, 136601 (2005).

33. Castellanos-Gomez, A. et al. Deterministic transfer of two-dimensional materials by all-dry viscoelastic stamping. 2D Mater. 1, 11002 (2014).

34. Kulakovskii, V. D. et al. Bose - Einstein Condensation of Exciton Polaritons in High Q Planar Microcavities with GaAs Quantum Wells. JETP Lett. 92, 595-599 (2010).

35. Glazov, M. M. et al. Intrinsic exciton-state mixing and nonlinear optical properties in transition metal dichalcogenide monolayers. Phys. Rev. B 95, 35311 (2017).

36. Wang, G. et al. Exciton states in monolayer $\mathrm{MoSe}_{2}$ : impact on interband transitions. 2D Mater. 2, 45005 (2015).

37. Schmutzler, J. et al. Nonlinear spectroscopy of exciton-polaritons in a GaAs-based microcavity. Phys. Rev. B - Condens. Matter Mater. Phys. 90, 1-6 (2014). 
38. Simon, H. J. \& Bloembergen, N. Second-Harmonic Light Generation in Crystals with Natural Optical Activity. Phys. Rev. 171, 1104-1114 (1968).

39. Jakubczyk, T. et al. Radiatively Limited Dephasing and Exciton Dynamics in MoSe 2 Monolayers Revealed with Four-Wave Mixing Microscopy. Nano Lett. 16, 5333-5339 (2016).

40. Cadiz, F. et al. Excitonic Linewidth Approaching the Homogeneous Limit in $\mathrm{MoS}_{2}$-Based van der Waals Heterostructures. Phys. Rev. X 7, 21026 (2017).

41. Wang, G. et al. Giant Enhancement of the Optical Second-Harmonic Emission of WSe Monolayers by Laser Excitation at Exciton Resonances. Phys. Rev. Lett. 114, 97403 (2015).

42. Leyder, C. et al. Observation of the optical spin Hall effect. Nature Physics 3, 628-631 (2007).

43. Maragkou, M. et al. Optical analogue of the spin Hall effect in a photonic cavity. 36, 10951097 (2011).

44. Baranowski, M. et al. Dark excitons and the elusive valley polarization in transition metal dichalcogenides. 2D Mater. 4, (2017).

45. Manni, F. et al. Spin-to-orbital angular momentum conversion in semiconductor microcavities. Phys. Rev. B 83, 241307 (2011).

46. Huang, S. et al. Topologically Protected Helical States in Minimally Twisted Bilayer Graphene. Phys. Rev. Lett. 121, 37702 (2018).

47. Klembt, S. et al. Exciton-polariton topological insulator. Nature 562 (2018).

48. Schmidt., D. et al. Tracking Dark Excitons with Exciton Polaritons in Semiconductor Microcavities. Phys. Rev. Lett. 122, 047403 (2019).

49. Tassone, F. and Yamamoto, Y. Exciton-exciton scattering dynamics in a semiconductor microcavity and stimulated scattering into polaritons. Phys. Rev. B 59, 10830 (1999).

50. Shahnazaryan, V., lorsh, I., Shelykh, I. A. and Kyriienko, O. Exciton-exciton interaction in transition-metal dichalcogenide monolayers. Phys. Rev. B 96, 115409 (2017).

\section{Acknowledgements}

C.S. acknowledges support by the ERC (Project unLiMIt-2D). The Würzburg group acknowledges support by the State of Bavaria. A.V.K. acknowledges the support from Westlake University (Project No. 041020100118). E.S. acknowledges support from the President of the Russian Federation for state support of young Russian scientists Grant No. MK-2839.2019.2 and the RFBR Grant No. 17-52-10006. S.K. acknowledges support by the EU (Marie Curie Project TOPOPOLIS). Q.Y. and S.T. acknowledge funding from NSF DMR-1838443 and DMR-1552220. M.M.G. acknowledges partial support from RFBR Project 17-02-00383. 